POS PROCEEDINGS

\title{
Evaluating multiloop Feynman integrals by differential equations
}

\author{
Vladimir A. Smirnov* \\ Nuclear Physics Institute of Moscow State University \\ E-mail: smirnovdtheory.sinp.msu.ru
}

\begin{abstract}
One year ago Henn suggested a new approach to solve differential equations for multiloop Feynman integrals. It is based on the notion of uniform transcendentality. A brief review of applications of this approach is presented.
\end{abstract}

Loops and Legs in Quantum Field Theory

27 April 2014 - 02 May 2014

Weimar, Germany

\footnotetext{
* Speaker.
} 


\section{Introduction}

Differential equations (DE) were, first, applied to evaluate multiloop Feynman integrals in [四, ㅁ]. Since then they were successfully applied in many papers. A crucial step in the development

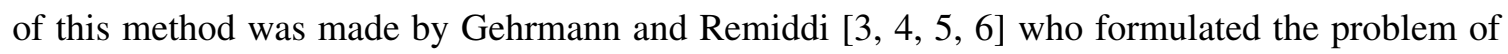
evaluating Feynman integrals at the level of master integrals which arise naturally when solving integration by part (IBP) relations [ [ $]$ ]. It is assumed that the master integrals are known, due to some algorithm of solving IBP relations. Taking derivatives of the master integrals with respect to given kinematic invariants and/or masses one can express these derivatives in terms of integrals of the given family with shifted indices. Then one expresses such integrals in terms of the master integrals with the help of the above mentioned algorithm and obtains a closed system of linear differential equations for the master integrals.

Let $x=\left(x_{1}, \ldots, x_{n}\right)$ be kinematical variables and $f=\left(f_{1}, \ldots, f_{N}\right)$ be the set of the master integrals revealed with some computer code. The DE take the following general form

$$
\partial_{i} f(\varepsilon, x)=A_{i}(\varepsilon, x) f(\varepsilon, x)
$$

where $\partial_{i}=\frac{\partial}{\partial x_{i}}$, each $A_{i}$ is an $N \times N$ matrix and $\varepsilon=(4-d) / 2$ is the parameter of dimensional regularization.

A next important step within the method of DE was made one year ago by Henn [ $[8]$ who conjectured that one can turn to a new basis by a linear transformation $f \rightarrow B f$ (resulting in $A_{i} \rightarrow$ $B^{-1} A_{i} B-B^{-1}\left(\partial_{i} B\right)$ ) such that the DE take the following canonical form

$$
\partial_{i} f(\varepsilon, x)=\varepsilon A_{i}(x) f(\varepsilon, x),
$$

where the elements of the new matrices $A_{i}(x)$ are independent of $\varepsilon$. In fact, it is also assumed that these elements are linear combinations of terms which are linear in $x$, or in variables which are introduced instead of $x$ with the typical goal to get rid of some square roots of polynomials in $x$ naturally arising for a given family of Feynman integrals. In the differential form, one has

$$
\mathrm{d} f(\varepsilon)=\varepsilon(\mathrm{d} \tilde{A}) f(x, \varepsilon),
$$

where $\tilde{A}=\sum_{i} \tilde{A}_{\alpha_{i}} \log \left(\alpha_{i}\right)$, where the arguments of the logarithms $\alpha_{i}$ are called letters.

The canonical DE $(\mathbb{L} .2)$ is much better than the primary version $(\mathbb{L} . \mathbb{d})$ because they can be solved in an expansion in $\varepsilon$ iteratively, with results typically expressed in terms of harmonic polylogarithms or multiple polylogarithms. Moreover, it is clear that such a solution will be manifestly uniformly transcendental (UT).

At the moment, there is no general algorithm to convert a given system of DE (페) into the canonical form $(\mathbb{L} 2)$. Rather, one uses various recipes. In simple situations, where master integrals can be expressed in terms of gamma functions at general $d$, one can adjust powers of the propagators in order to have the UT property. Moreover, one can directly use Feynman parameters to see desired properties of the element of the canonical basis. One more recipe is to replace propagators $1 /\left(-k^{2}\right)$ by delta functions $\delta\left(k^{2}\right)$ and use the experimental criterion that a given master integral is UT if the integral obtained after such a replacement is UT.

A brief review of applications of this new approach to solve DE is presented below. Then some perspectives of the approach are discussed in conclusion. 


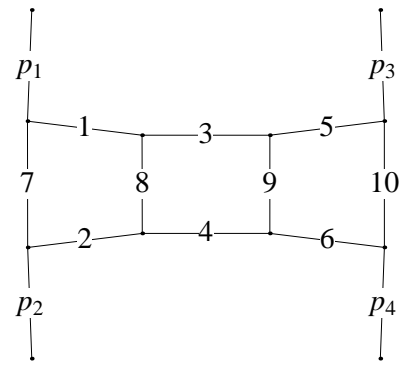

(A)

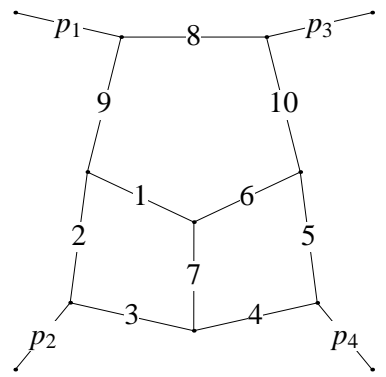

(E)

Figure 1: The triple box (A) and tennis court diagrams (E).

\section{A review of applications}

The first problem which was solved with the approach of [8] was the evaluation of planar three-loop massless diagrams with all the external legs on the light cone [Q]. These two families of Feynman integrals correspond to the ladder (i.e. triple box) and the tennis court graph shown in Fig. W. (The notation (A) and (E) for the three-loop four-point diagrams follows [ए]].) The first ten (of fifteen) indices of these integrals are associated with the the edges of these graphs and the last five correspond to irreducible numerators.

One year ago only two analytic results for non-trivial Feynman integrals associated with (A) and (E) were available: for the triple box (A) with the first ten indices equal to one without numer-

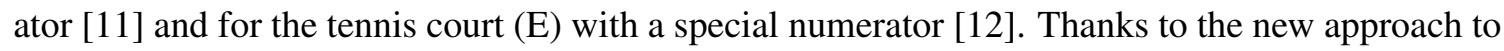
solve DE, all the corresponding master integrals were analytically evaluated in [Q] in an expansion in $\varepsilon$ up to weight six which is the typical weight for three-loop calculations. In both cases, the DE (ㅁ.2) take the following form

$$
\partial_{x} f(x, \varepsilon)=\varepsilon\left(\frac{a}{x}+\frac{b}{1+x}\right) f(x, \varepsilon) .
$$

where $x=t / s, s$ and $t$ are Mandelstam variables, $a$ and $b$ are $N \times N$ matrices with constant indices, with $N=26$ and $N=41$, respectively for (A) and (E). (These are particular cases of the KnizhnikZamolodchikov equation.) These equations are naturally solved in an expansion in $\varepsilon$, with results expressed [ $[$ ] in terms of harmonic polylogarithms. The natural boundary conditions came from the absence of singularities at $u \equiv-s-t \rightarrow 0$, i.e. $x \rightarrow-1$. They fix almost all the constants when solving DE so that the information which is missing after this is obtained from master integrals expressed in terms of gamma functions.

To evaluate all the massless non-planar three-loop four-point diagrams ((B), (C), (D), (F), (G), $(\mathrm{H})$, (I), in the terminology of [ए]] ) with all the legs on the light cone is a natural and challenging problem which will be, hopefully, solved within the approach of [ $[\mathbb{8}]$. As a warm-up, the diagram (C) shown in Fig. Z(a) was studied in [ए3] in the case where not only the last five (of fifteen) indices but also indices with the numbers 1,2,5,6 are non-positive. Such Feynman integrals are associated with the complete four-vertex graph $K_{4}$ consisting of four external vertices which are connected with each other by six lines - see Fig. $\mathbb{D}(\mathrm{b})$. 


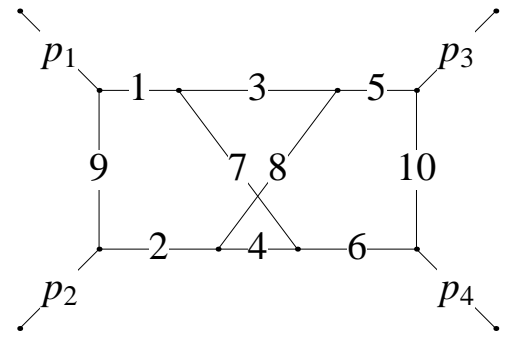

(a)

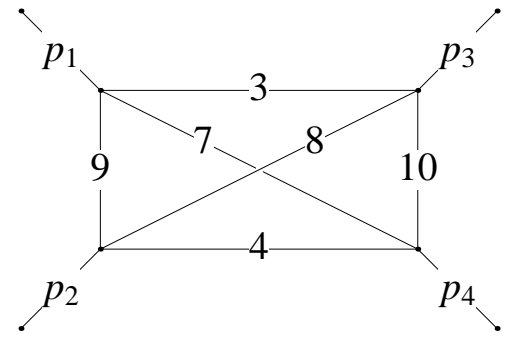

(b)

Figure 2: (a) Diagram (C). (b) The $K_{4}$ graph.

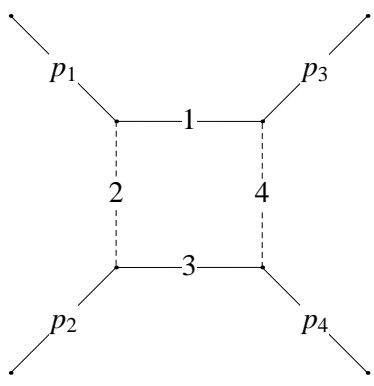

(1)

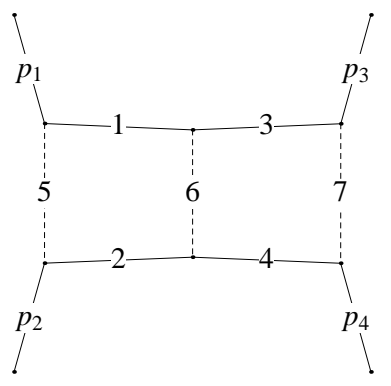

(2a)

Figure 3: One-loop and two-loop family of integrals (2a) for Bhabha scattering. Solid lines indicate massive propagators, and dashed lines massless ones.

The massless graph $K_{4}$ was recently discussed [1[4] in the context of the strategy of evaluating Feynman integrals by iterative integrations over Feynman parameters [ㄷ]], using multiple polylogarithms. ${ }^{1}$ It was claimed [प4] that the presence of $K_{4}$ as a subgraph means that the corresponding Feynman integral cannot be evaluated by iterative integrations.

In the family of Feynman integrals corresponding to $K_{4}$, there are three trivial master integrals expressed in terms of gamma functions and seven master integrals with six propagators. They were evaluated, by the approach of [[8], in terms of harmonic polylogarithms in [एँ3] in a Laurent expansion in $\varepsilon$ up to weight six.

One more example of application of the approach of $[\mathbb{[}]$ is the evaluation of one of the two two-loop planar families of Feynman integrals contributing to Bhabha scattering. They correspond to the graph shown in Fig. B (2a) and are labelled by seven indices for propagators and two indices for irreducible numerators. The external legs are on-shell, $p_{i}^{2}=m^{2}$, for $i=1,2,3,4$.

One year ago only for a small subset of the corresponding master integrals analytical results

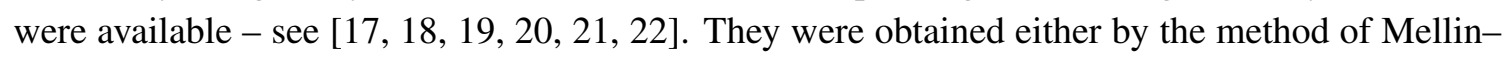
Barnes representation or by DE within the traditional approach. The evaluation of all the 23 master

\footnotetext{
${ }^{1}$ See, e.g., [[0]] for applications of this strategy.
} 
integrals was performed in [23]. After introducing new variables

$$
\frac{-s}{m^{2}}=\frac{(1-x)^{2}}{x}, \quad \frac{-t}{m^{2}}=\frac{(1-y)^{2}}{y},
$$

(to get rid of square roots) it was possible to arrive at canonical DE ([L.3]) where the letters of the alphabet in all but one master integral are $\{x, 1 \pm x, y, 1 \pm y, x+y, 1+x y\}$ while four much more complicated letters are present in one of the master integrals. Results for the master integrals were expressed in terms of multiple polylogarithms - see [23]].

Recently all the master integrals for two-loop massless double-box diagrams with two legs off the light cone (i.e. with $p_{1}^{2}=0, p_{2}^{2}=0$ and $p_{3}^{2}=M_{3}^{2}, p_{4}^{2}=M_{4}^{2}$ ) were analytically evaluated in [24, 25, [26, [27]. They are essential ingredients of NNLO QCD corrections to the production of two off-shell vector bosons in hadron collisions. There are three planar and three nonplanar families of the Feynman integrals which have at most seven positive indices, with the total number of indices equal to nine. In [24, 26] the equal mass case, $M_{3}=M_{4}$, was considered. In [24], the planar master integrals were evaluated by DE in the traditional approach, while in [25, [26, 27] the approach of [8] was applied.

For the planar diagrams and for two families of nonplanar diagrams, new variables were introduced by

$$
\frac{s}{M_{3}^{2}}=(1+x)(1+x y), \frac{t}{M_{3}^{2}}=-x z, \frac{M_{4}^{2}}{M_{3}^{2}}=x^{2} y
$$

and for one of the nonplanar families by

$$
\begin{aligned}
s & =M^{2}(1+x)^{2}, \quad t=-M^{2} x((1+y)(1+x y)-2 z y(1+x)), \\
M_{3}^{2} & =M^{2} x^{2}\left(1-y^{2}\right), M_{4}^{2}=M^{2}\left(1-x^{2} y^{2}\right)
\end{aligned}
$$

These are letters for the planar families: $\{x, y, z, 1+x, 1-y, 1-z, 1+x y, z-y, 1+y(1+x)-z, x y+$ $z, 1+x(1+y-z), 1+x z, 1+y-z, z+x(z-y)+x y z, z-y+y z+x y z\}$.

For two non-planar families, one has $\{x, 1+x, 1-y, y, 1+x y, 1+x(1+y-z), 1-z, y-z, 1+y-$ $\left.z, 1+y+x y-z, z,-y+z, x y+z, 1+x+x y-x z, 1+x z, 1+y+2 x y-z+x^{2} y z, z-y(1-z-x z)\right\}$ and, for the third non-planar family, $\{x, 1+x, 1-y, y, 1+y, 1-x y, 1+x y, 1-y(1-2 z), 1+y-2 y z, 1-$ $x y^{2}-y(1-x-2 z+2 x z), 1-x y(1-2 z), 1+x(y-2 y z), 1+x y^{2}-(1+x) y(1-2 z), 1-z, z, 1+y-$ $\left.2 y z,(1+y)(1+x y)-2 z y(1+x), 1-y+2 y z, 1-x y^{2}+(1-x) y(1-2 z)\right\}$.

Although there is a quadratic dependence on some variables in the letters, it was possible to integrate DE in terms of multiple polylogarithms. The natural strategy to achieve this was to postpone the integration over variables with a quadratic dependence to the very end. The results of [25, 27] are in agreement, in the equal mass case, with results of [24, 26] .

\section{Conclusion}

It turned out that, with the approach of [ [8]], it became possible to evaluate master integrals for quite complicated families of Feynman integrals which were not feasible before. I believe that many other problems will be solved with the help of this approach, in particular, the nonplanar diagrams (B), (C),... mentioned in the previous section, the second type of planar and the 
non-planar type of on-shell massive double boxes relevant to Bhabha scattering and many other problems.

It is also possible to apply this approach to single-scale diagrams. This was demonstrated in [[13] on the example of a three-loop non-planar master integral contributing to quark and gluon form-factors which was previously evaluated in [ㄹ, [29, [3] ]. The underlying idea is to introduce one more scale. On the one hand, this complicates the situation. On the other hand, one obtains the possibility to apply the power of the method of DE. I am confident that new results will be obtained also with the help of this strategy.

A subtle point of the approach of [ [ $]$ ] is the transition from a primary basis of the master integrals to a canonical basis. To construct an algorithm applicable at least in some specific situation (for example, the massless case with two scales), is a very natural and important mathematical problem. ${ }^{2}$

\section{References}

[1] A. V. Kotikov, Phys. Lett. B 254 (1991) 158.

[2] A. V. Kotikov, Phys. Lett. B 267 (1991) 123.

[3] E. Remiddi, Nuovo Cim. A 110 (1997) 1435 [hep-th/9711188].

[4] T. Gehrmann and E. Remiddi, Nucl. Phys. B 580 (2000) 485 [hep-ph/9912329].

[5] T. Gehrmann and E. Remiddi, Nucl. Phys. B 601 (2001) 248 [hep-ph/0008287].

[6] T. Gehrmann and E. Remiddi, Nucl. Phys. B 601 (2001) 287 [hep-ph/0101124].

[7] K. G. Chetyrkin and F. V. Tkachov, Nucl. Phys. B 192 (1981) 159.

[8] J. M. Henn, Phys. Rev. Lett. 110 (2013) 25, 251601 [arXiv:1304.1806 [hep-th]].

[9] J. M. Henn, A. V. Smirnov and V. A. Smirnov, JHEP 1307 (2013) 128 [arXiv:1306.2799 [hep-th]].

[10] Z. Bern, J. J. Carrasco, L. J. Dixon, H. Johansson, D. A. Kosower and R. Roiban, Phys. Rev. Lett. 98 (2007) 161303 [hep-th/0702112].

[11] V. A. Smirnov, Phys. Lett. B 567 (2003) 193 [hep-ph/0305142].

[12] Z. Bern, L. J. Dixon and V. A. Smirnov, Phys. Rev. D 72 (2005) 085001 [hep-th/0505205].

[13] J. M. Henn, A. V. Smirnov and V. A. Smirnov, JHEP 1403 (2014) 088 [arXiv:1312.2588 [hep-th]].

[14] C. Bogner and M. Lüders, arXiv:1302.6215 [hep-ph].

[15] F. Brown, Commun. Math. Phys. 287 (2009) 925 [arXiv:0804.1660 [math.AG]].

[16] E. Panzer, Nuclear Physics, B 874 (2013), 567 [arXiv:1305.2161 [hep-th]].

[17] V. A. Smirnov, Phys. Lett. B 524 (2002) 129 [hep-ph/0111160].

[18] G. Heinrich and V. A. Smirnov, Phys. Lett. B 598 (2004) 55 [hep-ph/0406053].

[19] M. Czakon, J. Gluza and T. Riemann, Phys. Rev. D 71 (2005) 073009 [hep-ph/0412164].

[20] M. Czakon, J. Gluza and T. Riemann, Acta Phys. Polon. B 36 (2005) 3319 [hep-ph/0511187].

\footnotetext{
${ }^{2} \mathrm{~A}$ step towards solving it was made in [B] $]$ where a relatively simple situation (with a linear dependence of the matrix $A$ in $(\mathbb{L} \mathbb{C})$ on $\varepsilon$ ) was considered.
} 
[21] M. Czakon, J. Gluza and T. Riemann, Nucl. Phys. B 751 (2006) 1 [hep-ph/0604101].

[22] M. Czakon, J. Gluza, K. Kajda and T. Riemann, Nucl. Phys. Proc. Suppl. 157 (2006) 16 [hep-ph/0602102].

[23] J. M. Henn and V. A. Smirnov, JHEP 1311 (2013) 041 [arXiv:1307.4083].

[24] T. Gehrmann, L. Tancredi and E. Weihs, JHEP 1308 (2013) 070 [arXiv:1306.6344, arXiv:1306.6344 [hep-ph]].

[25] J. M. Henn, K. Melnikov and V. A. Smirnov, JHEP 1405 (2014) 090 [arXiv:1402.7078 [hep-ph]].

[26] T. Gehrmann, A. von Manteuffel, L. Tancredi and E. Weihs, JHEP 1406 (2014) 032 [arXiv:1404.4853 [hep-ph]].

[27] F. Caola, J. M. Henn, K. Melnikov and V. A. Smirnov, arXiv:1404.5590 [hep-ph].

[28] G. Heinrich, T. Huber, D. A. Kosower and V. A. Smirnov, Phys. Lett. B 678 (2009) 359 [arXiv:0902.3512 [hep-ph]].

[29] P. A. Baikov, K. G. Chetyrkin, A. V. Smirnov, V. A. Smirnov and M. Steinhauser, Phys. Rev. Lett. 102 (2009) 212002 [arXiv:0902.3519 [hep-ph]].

[30] R. N. Lee and V. A. Smirnov, JHEP 1102 (2011) 102 [arXiv:1010.1334 [hep-ph]].

[31] M. Argeri, S. Di Vita, P. Mastrolia, E. Mirabella, J. Schlenk, U. Schubert and L. Tancredi, JHEP 1403 (2014) 082 [arXiv:1401.2979 [hep-ph]]. 